\title{
Anabases
}

ANABASES Traditions et réceptions de l'Antiquité

$9 \mid 2009$

Varia

\section{Des fouilles aux tranchées. Les jalons de Déchelette}

\section{Serge Lewuillon}

\section{OpenEdition}

Journals

Édition électronique

URL : http://journals.openedition.org/anabases/495

DOI : 10.4000/anabases.495

ISSN : 2256-9421

\section{Éditeur}

E.R.A.S.M.E.

\section{Édition imprimée}

Date de publication : 1 mars 2009

Pagination : 249-260

ISSN : 1774-4296

\section{Référence électronique}

Serge Lewuillon, « Des fouilles aux tranchées. Les jalons de Déchelette », Anabases [En ligne], 9| 2009, mis en ligne le 01 mars 2012, consulté le 20 octobre 2019. URL : http://journals.openedition.org/ anabases/495 ; DOI : 10.4000/anabases.495 


\section{Des fouilles aux tranchées. Les jalons de Déchelette}

Serge LeWUillon

\section{Archéologue malgré eux}

Contrairement À D’AUtres Pionniers de l'archéologie française, Joseph Déchelette reste largement méconnu, même dans le monde savant. Tout au plus sa silhouette apparaitelle au visiteur de Bibracte comme celle d'un des inventeurs de l'oppidum. Pour le reste, Déchelette est l'homme d'un seul livre, mais quel livre: le Manuel d'archéologie préhistorique, celtique et gallo-romaine, considéré par beaucoup comme la cheville ouvrière de la protohistoire française ${ }^{1}$. On le cite, plus par révérence que par référence, mais sans s'appesantir sur son rôle dans la constitution de l'archéologie européenne. Ce relatif détachement vient malheureusement de ce que Joseph Déchelette a fait une carrière scientifique en marge des institutions, avec pour seul titre professionnel celui de conservateur du musée de Roanne. Ni cette position, ni son affiliation à des sociétés savantes plus ou moins importantes ne sont cependant à la mesure de la tâche qu'il a accomplie.

En dépit de son esprit novateur, Déchelette reste foncièrement un homme du $\mathrm{XIX}^{\mathrm{e}}$ siècle. Les bornes de son existence sont le second Empire, dont il reçut le goût des antiquités nationales, et la Grande Guerre, où il laissa la vie. Bien que sur le moment, sa disparition ait créé un choc dans la communauté archéologique internationale, l'hommage de ses pairs apparut bientôt tempéré par des réserves sur la portée de son travail, sinon sur sa stature d'homme de science. En fait, Déchelette ignorait l'orgueil: arc-bouté sur sa province, où il avait fait son apprentissage et dans laquelle s'enracinait son expérience d'archéologue, il avait toujours résisté aux tentations que l'on dit

1 J. DéChelette, Manuel d'archéologie préhistorique, celtique et gallo-romaine, Paris, 19081914 ( 2 tomes en 6 vol., dont 2 vol. d'appendices). 
parisiennes. Il avait mis son ambition archéologique dans un ouvrage de synthèse, ainsi que dans un projet historiographique plus vaste encore. De son point de vue, le Manuel serait un chef-d'œuvre au sens propre: non seulement le gage ultime donné à la communauté scientifique, mais aussi l'état des lieux de la protohistoire, qui légitimerait ses investigations sur la civilisation européenne des origines. Pour son malheur, Déchelette passait aux yeux de ses pairs pour un notable, tout empreint de «sociabilité érudite " provinciale, qui se serait arrogé la mission de régenter l'archéologie européenne. Cette prétention lui a sans doute coûté une reconnaissance mieux établie. De plus, Déchelette ne disparut pas tout seul: au lendemain de la guerre, c'est une école archéologique considérable qui s'est évanouie, ce groupe de Saint-Germain-en-Laye qui prônait de recombiner l'histoire, la sociologie et l'ethnographie au sein d'une vaste synthèse comparatiste. L'archéologie française étant retournée à sa routine pour quelques décennies, le Manuel a simplement couru sur son erre, tandis que la réputation de son auteur jaunissait sans s'effacer totalement.

\section{Le métier d'archéologue}

La biographie ordinaire de Déchelette est écrite ${ }^{2}$ : on sait presque tout de l'homme, tout au moins ce que les convenances d'une époque en ont laissé filtrer. On doit à sa veuve le collationnement de la correspondance scientifique de son mari : il en émane un portrait officiel, dont il ne faut attendre aucune surprise, nulle fausse note. En revanche, l'étude de ses archives révèle que Déchelette n'établissait pas de distinction entre sa vie professionnelle et ses activités archéologiques. Du point de vue du chercheur, cet angle de vue peut s'avérer utile à l'interprétation de son projet scientifique, qui fut long et tortueux, mais rationnel d'un bout à l'autre. L'organisation de son temps, de ses voyages, le choix de ses destinations et de ses correspondants, la planification de ses sujets d'étude et de ses travaux, ses investissements et l'enrichissement de sa bibliothèque, tout contribue à éclairer sa formation et ses aspirations scientifiques d'un jour nouveau - mieux sans doute que ne le font les brouillons de ses livres, qu'il vaudrait mieux laisser à leur aspect définitivement lacunaire.

Déchelette s'était tourné à l'origine vers l'étude du patrimoine monumental plutôt que vers l'archéologie proprement dite, en un temps et dans un milieu où cela n'allait pas de soi. D'abord, il n'existait pas de formation spécifique à ce métier, qui n'en était même pas un officiellement. Par ailleurs, les goûts de Déchelette le portaient irrésistiblement vers les archives. Il avait d'abord songé à l'École des Chartes, avant

2 M.-S. BinÉTRuy, De l'Art roman à la Préhistoire, des sociétés locales à l'Institut, itinéraire de Joseph Déchelette, Lyon, 1994. Fr. Déchelette éd., Livre d'or de J. Déchelette. Centenaire 1862-1962. Roanne, 1962. S. Lewuillon, "Joseph Déchelette, un archéologue européen”, in Ph. SÉNÉCHAL dir., Dictionnaire des historiens de l'art en France au XIX siècle, Paris, INHA (à paraître en 2009). 
d'être contraint d'y renoncer pour se consacrer à l'entreprise familiale de tissage. Néanmoins, il eut la chance de fréquenter à ses débuts quelques personnages marquants auprès desquels il puisa sa sensibilité au patrimoine et à l'histoire et qui le confirmèrent dans sa vocation de chercheur. Parmi ceux-ci, son oncle Jacques-Gabriel Bulliot, dans le négoce comme lui, qui s'était fait connaitre comme l'archéologue du Mont-Beuvray (identifié pour la première fois comme l'antique Bibracte citée par César). L'ayant associé à ses recherches, Bulliot lui fournit involontairement un sujet de recherches historiques de grande portée ${ }^{3}$. D’un autre côté, le milieu provincial où Déchelette avait fait ses débuts n'avait pas que des aspects négatifs. C'est au sein d'une société savante de bonne réputation, la Diana, qu'il affirma sa prédilection pour le Moyen Âge et s'initia à l'architecture religieuse. Ce choix ne fut pas sans conséquence sur son évolution intellectuelle, car il l'obligea à diverses mises au point méthodologiques qu'il n'aurait pu trouver chez les premiers maîtres de l'archéologie monumentale comme de Caumont ou Viollet-le-Duc. Il y gagna une tournure d'esprit qui conciliait l'histoire de l'art et l'archéologie, pressentant derrière leur nécessaire combinaison la dynamique des filiations et des transitions historiques. Dans son premier mémoire sur les peintures murales du Forez, Déchelette évoque les règles d'une méthode qui fera la fortune de son Manuel:

Les fiches que j'ai réunies étant assez nombreuses - environ trois cents - l'idée m'est venue de dresser une bibliographie analytique et raisonnée de tous les travaux parus sur les peintures murales de France. Je trouve utile et intéressant de relier de temps en temps par un lien bibliographique les travaux des provinciaux entre eux sans oublier les Parisiens ${ }^{4} \ldots$

Ensuite, c'est en rédigeant les notices de l'Inventaire des Richesses d'art de la France et dans les premiers classements qui s'ensuivirent que Déchelette fit l'expérience de la typologie. C'est enfin dans le classement de plusieurs bibliothèques et fonds d'archives régionaux qu'il recueillit avec un certain opportunisme qu'il comprit le poids des bibliographies. La méthode de Déchelette tient tout entière dans le développement de ses recherches selon ces trois axes.

\section{L'hypothèse de Stradonice}

Ayant pris très au sérieux son rôle de conservateur adjoint au musée de Roanne, Déchelette eut l'heureuse intuition d'y étudier une série de vases peints laissés là à leur incognito.

3 J. Déchelette, Les Fouilles du mont Beuvray de 1897 à 1901, compte rendu suivi de l'inventaire général des monnaies recueillies au Beuvray et du Hradischt de Stradonice en Bohême, étude d'archéologie comparée, Paris, 1904 [Extrait des Mémoires de la Société éduenne, Nouvelle série, t. XXII].

4 E. Brassart, J. Déchelette dir., Peintures murales du Moyen Âge et de la Renaissance en Forez, Montbrison, 1900. 
Mû par un systématisme dont il ferait pour ainsi dire sa signature, il mit au point à cette occasion les principes d'analyse céramologique qui devaient par la suite lui ouvrir bien des portes 5 . La publication de ce matériel attira sur lui l'attention de Salomon Reinach, directeur du musée des Antiquités nationales de Saint-Germain-en-Laye, qui l'invita à collaborer avec Henri Hubert. Jusqu'alors, Déchelette n'avait pas éprouvé de curiosité excessive pour la protohistoire: malgré sa collaboration avec Bulliot, sa conception du terrain demeurait très classique et essentiellement logistique. En revanche, il continuait de mettre dans le classement et la publication des monuments du passé toute sa passion d'archiviste contrarié. Pour l'heure, il ne s'agissait encore que de simples objets abandonnés dans des réserves, mais bientôt, il appliquerait sa méthode analytique à des structures d'une toute autre envergure. Cela se remarqua au congrès d'archéologie préhistorique d'Autun en 1907, à l'occasion duquel il définit de façon synthétique sa conception des oppida. C'est justement la céramique qui avait joué un rôle essentiel dans cet essai. De passage à Berlin en 1899, Déchelette y avait remarqué un fragment de céramique peinte comme il en avait vu au Mont-Beuvray. Sur recommandation, il se rendit au musée national de Prague, où il en découvrit des vitrines entières provenant de l'oppidum tout proche de Stradonice. Il y avait là non seulement des céramiques, mais également du matériel de métal, d'émail et de verre en tout point comparable à celui de Bibracte. Par ailleurs, la même parenté semblait pouvoir être établie entre les faciès monétaires de Bourgogne et de Bohème. À cette occasion, Déchelette entama une relation suivie avec le conservateur du musée de Prague, qui venait d'éditer une étude de l'oppidum de Stradonice et de ses origines. Le savant français jugea ce livre si intéressant pour son propos qu'il se mit à l'étude du tchèque et le traduisit ${ }^{6}$.

Interprétant les similitudes archéologiques entre les matériels de Bibracte et de Stradonice comme la marque d'un commerce établi entre deux communautés au cours du second âge du Fer, Déchelette posait les jalons d'une théorie historique qui devait être reprise jusqu'à notre époque: selon lui, les deux oppida devaient être considérés comme les témoins d'une ancienne unité culturelle, assimilable à l'une des premières civilisations européennes: celle des Celtes historiques ou, en termes archéologiques, la culture de La Tène. Cette thèse revêtait une grande importance pour l'archéologie européenne, car elle offrait un début de réponse à la question de l'identité des peuples protohistoriques. Surtout, elle emportait de lourdes conséquences pour les recherches archéologiques en cours: l'étude des sociétés préhistoriques devrait désormais renoncer aux habituels principes nationalistes pour s'appuyer sur la chronologie archéologique. La tâche prioritaire des chercheurs consistait donc à établir une chronologie concordante entre les différentes

5 J. Déchelette, Les Vases céramiques ornés de la Gaule romaine: Narbonnaise, Aquitaine et Lyonnaise, Le Blanc-Mesnil, 1979 [fac-sim. de l'éd. de Paris, 1904].

6 J. Déchelette, Le Hradischt de Stradonic en Bohême et les fouilles de Bibracte, étude d'archéologie comparée, Mâcon, 1901 [Extrait du Congrès archéologique de Mâcon, juin 1899]. 


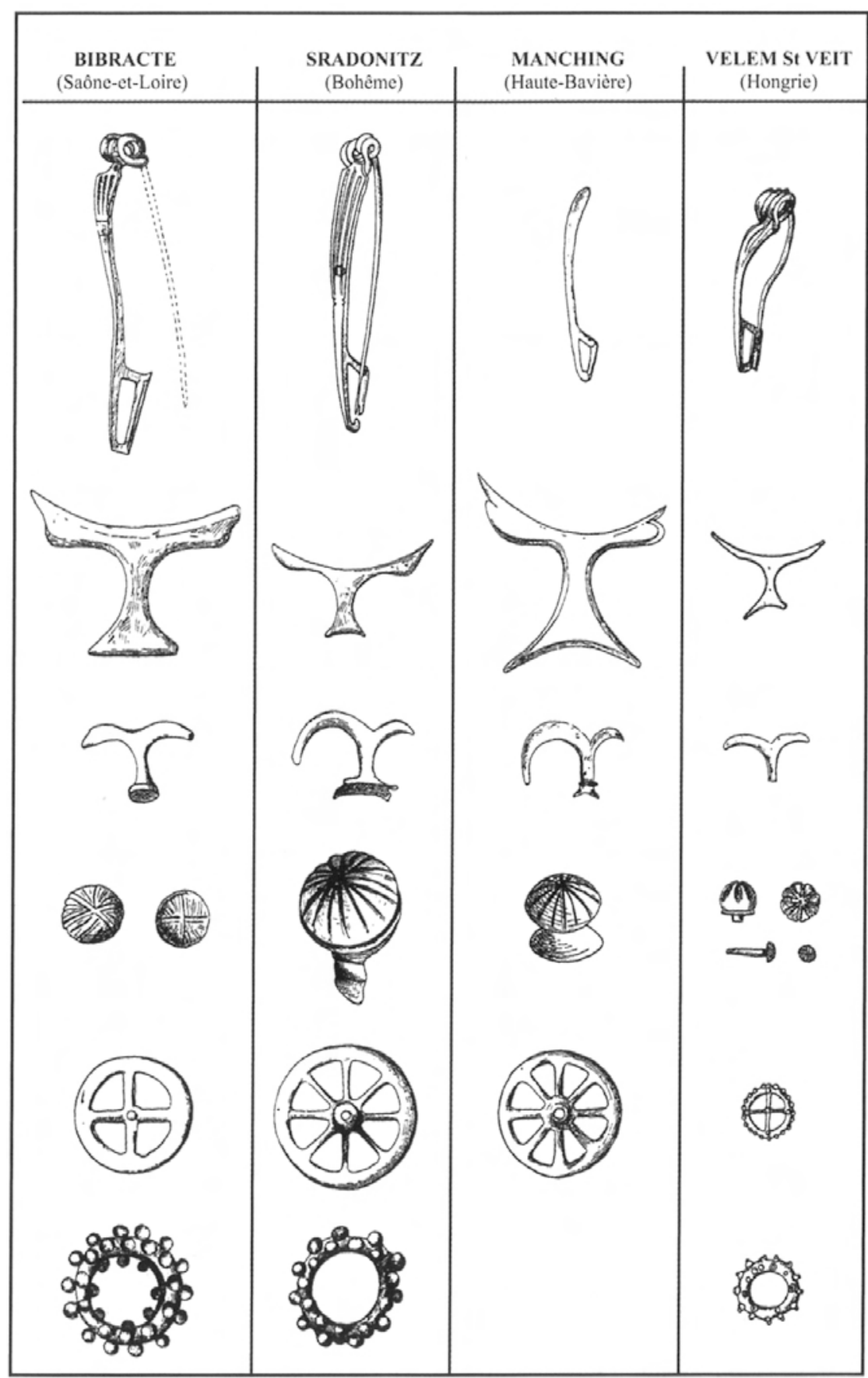

Fig. 1: «Menus objets semblables trouvés dans quatre stations de La Tène III »(Manuel... IV, p. 477, fig. 404) 
cultures archéologiques d'Europe par une patiente comparaison des matériels céramiques et métalliques issus des fouilles et gisant dans les musées. Telle était la loi fondamentale de l'archéologie, inlassablement exposée au fil des pages du Manuel. Elle impliquait entre autres que la protohistoire serait européenne ou ne serait pas. De nos jours, ce paradigme européen a beaucoup marqué les esprits, allant parfois jusqu'à prendre une acception indue et disproportionnée. L'idée d'une préfiguration celtique de la civilisation européenne s'installa durablement dans l'historiographie du vieux continent pour culminer à partir du début des années 1990, au moment où la construction européenne était profondément marquée par la réunification allemande. Du point de vue archéologique, la célèbre exposition I Celti de Venise en constitua le point d'orgue.

En son temps, la découverte de Stradonice avait agi sur Déchelette comme une révélation. Dès ce moment, il n'eut plus d'autre souci que de perfectionner sa connaissance des sites archéologiques, s'adonnant exclusivement à l'étude des âges du Fer, cette période cruciale du premier millénaire avant notre ère, au cours de laquelle s'était déclarée l'identité celtique. Cette question devenait si prégnante que, pour en connaître le fin mot, Déchelette renonça à la gestion de l'entreprise familiale, tout en demeurant marqué par les leçons de son ancien métier. Persuadé que la culture marche toujours sur les traces de l'économie et ne se maintient qu'au prix des échanges, il attachait un grand prix aux causes matérielles qui conduisent les hommes à s'associer plutôt qu'aux dispositions psychologiques qui les poussent à se distinguer les uns des autres.

Dans cet ordre d'idées, Déchelette rejetait tout particulièrement le nationalisme, comme le montre l'épisode du livre de Pič. Malgré son intérêt pour cet ouvrage, la traduction de Déchelette n'en rapportait pas fidèlement toute la teneur ${ }^{7}$. Des désaccords subsistaient à propos d'une interprétation jugée excessivement nationaliste de certains pans de l'histoire tchèque antique, entraînant des divergences de vue sur la chronologie protohistorique. Comme on ne connaît en France que la traduction de Déchelette, il a paru intéressant de reconnaître les principes l'ayant guidé dans son interprétation, en comparant les versions tchèque et française de l'ouvrage, ainsi que le contexte archéologique et historiographique de chaque pays ${ }^{8}$. Les premiers résultats de cette étude confirment qu'il demeure instructif de décrypter les présupposés idéologiques qui n'ont cessé de gauchir la référence aux antiquités nationales dans tous les pays d'Europe depuis le XIX ${ }^{\mathrm{e}}$ siècle.

\footnotetext{
7 J. PIč, Le Hradischt de Stradonitz en Bohême (ouvrage traduit du tchèque par Joseph Déchelette), Leipzig, 1906.

$8 \quad$ Projet auquel Gilles Pierrevelcin (Université de Strasbourg, Bibracte, doctorant à l'université Charles de Prague), Pavel Sankot (conservateur actuel du Musée National de Prague) et moi-même sommes associés.
} 


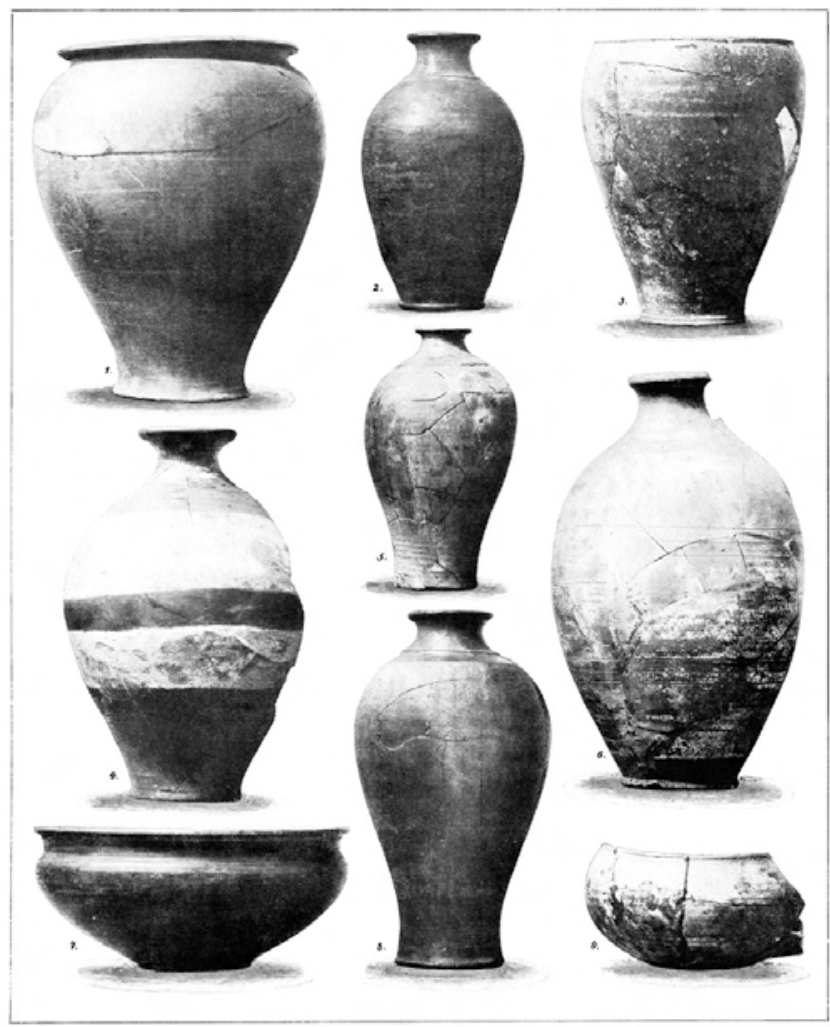

Fig. 2: La céramique du Hradischt de Stradonice dans la monographie tchèque9

\section{Discours de la méthode}

L'hypothèse de Stradonice ne se limitait pas à la Bourgogne ni à la Bohème, mais concernait les différentes contrées européennes où l'on rencontrait des conditions environnementales et archéologiques similaires. Mettant à profit les contacts qu'il avait noués au cours de ses voyages d'affaires, Déchelette mûrit peu à peu une stratégie discrète qui devait le placer au centre du cercle des protohistoriens. Il rapportait tout dans ses carnets de voyage, dont il tirait des notes variées et des statistiques élémentaires. D'observateur, il devint ainsi presque naturellement l'ordonnateur d'un ensemble de connaissances éparpillées qui ne demandaient, pour faire sens, que d'être structurées. C'est en raison de l'ampleur de sa méthode, mais aussi de la hardiesse de ses hypothèses et de l'identité de vue entre Déchelette et l'école de Saint-Germain que Salomon

9 J. PIč, Čechy na úsvitě dějin. Na základě praehistorické sbírky Musea království českého a pramenů dějepisných, Prague, 1902-1903, vol. 2, pl. L. 
Reinach proposa au savant roannais la rédaction d'un manuel qui ferait le point sur l'archéologie européenne. Déchelette se laissa convaincre et entreprit de déverser son immense documentation dans un ouvrage ambitieux, dont le plan allait de la préhistoire à l'époque gallo-romaine.

Le principe de cette rédaction était largement empirique. Aussi, ce que Déchelette apprit de la préhistoire - comme ce qu'il ne parvint pas à en apprendre - lui fut d'une grande utilité pour analyser les âges du Bronze et du Fer. Le premier volume du Manuel embrassait une science qui dépassait manifestement les connaissances livresques de l'autodidacte: sa rencontre avec la préhistoire ne fut donc pas très heureuse et n'aboutit qu'à générer un malentendu persistant avec les représentants de cette discipline (en particulier la SPF). Au delà de quelques réflexions sur la portée de l'art préhistorique, Déchelette s'attachait surtout à classer le matériel le plus méthodiquement possible, notamment pour le néolithique. Cette méthode donna surtout des résultats dans l'étude des mégalithes, ainsi que dans celle des céramiques. Mais au moment même où il avait à sa portée le principe de la périodisation, Déchelette n'arrivait pas à trancher entre deux principes majeurs de la préhistoire: la conception fonctionnelle et la conception culturelle. S'abstenant de raisonner en termes de "races " (préhistoriques), il choisit d'élaborer sa propre théorie de l'évolution des sociétés, portant l'accent sur l'évolution culturelle de groupes humains déjà constitués plutôt que les migrations.

Paradoxalement, ce choix de la "nature " (l'identité étant une donnée) contre la " culture " (la diffusion de la donnée originelle étant un processus) devait être relativisé dans les tomes suivants du Manuel. C'est que, chemin faisant, Déchelette avait conforté son " hypothèse de Stradonice ", c'est-à-dire l'existence d'une communauté culturelle protohistorique rayonnant à partir de l'Europe moyenne. Cette conception est résumée par une planche du Manuel peu spectaculaire, mais lourde de sens, établissant un parallèle entre plusieurs groupes d'objets provenant de quelques hauts lieux de l'archéologie celtique, comme une préfiguration de la "civilisation des oppida ". Mais cet élargissement ethnologique, géographique et chronologique exigeait une meilleure intégration des recherches portant sur toute l'aire présumée des oppida: l'enquête protohistorique de Déchelette prit l'allure d'une entreprise collective, fondée sur un réseau international de correspondants. En réponse à la collecte des données, Déchelette apportait à ses collègues un contexte chronologique et culturel qui offrait un sens nouveau à leurs découvertes, tandis que lui-même y trouvait de quoi fournir ses propres tableaux. Enfin, d'une entreprise qui renforçait la science française vis-à-vis des travaux germaniques, il pouvait espérer en tirer personnellement avantage: par l'édition du Manuel, où toute cette expérience était consignée, il s'affirmait dans le monde savant en y imprimant sa marque et en y imposant sa méthode.

Il démontra tout d'abord la nécessité d'établir une chronologie de portée générale et concordante avec les autres expériences européennes. L'exigence d'uniformisation augmentait avec la diversification des cultures matérielles, du vocabulaire et des références archéologiques. C'est ainsi que Déchelette prit conscience, non sans esprit critique, de la prééminence de l'approche taxonomique. Il recueillit le système de Montélius 
pour la chronologie relative et ceux de Hoernes, Tischler, Reinecke, Viollier, etc. pour la chronologie absolue, afin d'élaborer son propre tableau synoptique. Ce programme le conduisit à s'intéresser de plus près aux populations établies dans l'aire celtique, c'està-dire à poursuivre l'identification et la localisation des ethnies citées par les auteurs classiques, puis à reconnaître les relations de parenté qu'elles entretenaient entre elles. À la lecture des deux tomes consacrés à l'âge du Fer, on est frappé de l'amplification de la problématique mise en œuvre, comme si l'auteur, butant pour la première fois sur une civilisation moderne et authentique, prenait soudain conscience de la profondeur de l'histoire. Nul doute qu'en cette occasion, le Manuel se soit hissé au niveau de l'analyse historique, au sens où l'entendait Reinach.

L'itinéraire de Déchelette prit alors une nouvelle direction. Sa collaboration avec Henri Hubert l'avait déjà entrâné sur les chemins du comparatisme et l'avait sensibilisé à la sociologie: plusieurs de ses études s'en ressentent, comme l'analyse des broches étrusques et gauloises d'Italie du Nord ${ }^{10}$. Mieux encore, Reinach avait mis l'archéologue en relation avec le cercle des "nouveaux historiens", recommandant l'inventeur de la céramique gauloise et de la civilisation des oppida à Henri Berr pour sa Revue de Synthèse ${ }^{11}$. Pour diverses raisons idéologiques, mais aussi en raison des circonstances tragiques de la Grande Guerre, la portée de l'archéologie comparée en général et la contribution qu'y a apportée Déchelette en particulier sont sorties affaiblies de cette période. Cette tentative de refondation de l'archéologie a cependant laissé des traces dans les archives. Dans la seconde moitié du XIX ${ }^{\mathrm{e}}$ siècle, des archéologues aussi savants qu'artistes, tels que de Ring, Chantre, Flouest ou Cournault s'étaient mis à courir les musées d'Europe, afin de rassembler, d'agencer, de mettre en regard les unes des autres les pièces les plus variées propres à témoigner des similitudes ou des divergences dans les cultures matérielles. Ils pressentaient que le mobilier des cultures protohistoriques ne livrerait tout son sens que dans le dévoilement de sa dimension ethnographique, grâce à de larges comparaisons interculturelles. Il restait à tirer parti de ce musée de papier, qui laissait présager l'avènement d'une archéologie d'ouverture.

À Saint-Germain-en-Laye, Hubert était le dépositaire d'une masse considérable de documents iconographiques originaux, tandis qu'à Roanne, Déchelette faisait de même, acquérant la plupart des albums illustrés parus depuis la première moitié du $\mathrm{XIX}^{\mathrm{e}}$ siècle. L'étude précise des collections iconographiques qu'il a rassemblées dans sa bibliothèque de Roanne est donc aujourd'hui du plus haut intérêt pour saisir à

10 J. DéchelEtte, "Broches en fer d'époque gauloise servant de monnaies primitives. Étude sur les origines de la drachme et de l'obole", in La collection Millon. Antiquités prébistoriques et gallo-romaines, Paris, 1913, p. 191-243; fig. 35, p. 201 (= Revue numismatique, 1911, p. 1-59). Aussi Manuel d'archéologique préhistorique et celtique. III: Premier âge du Fer. Époque de Hallstatt, Paris, 1927, p. 285-295 ; Manuel d'archéologique prébistorique et celtique, IV: Second âge du Fer. Époque de La Tène, Paris, 1927, p. 918-934.

11 J. DÉCHeLETTE, "L'archéologie celtique en Europe". Revue de Synthèse historique 7 (1901), p. 1-29. 
quel point le travail de Déchelette était fondé sur le comparatisme. Depuis plusieurs années, une enquête a été entreprise dans ce sens avec le concours de l'INHA dans le cadre du programme AREA (Archives de l'archéologie européenne) ${ }^{12}$. Dans la perspective du centenaire de la disparition de Déchelette, on entretient le projet d'une exposition sur l'iconographie archéologique en France: «La bibliothèque de Joseph Déchelette à Roanne: un panorama de l'iconographie archéologique au $\mathrm{XIX}^{\mathrm{e}}$ siècle $^{13}$ ».

\section{La tentation de l'histoire}

À lire ses notes et sa correspondance, tout indique que Déchelette était en plein accord avec les tenants de l'histoire nouvelle. Cette mouvance comptait des partisans résolus à Saint-Germain-en-Laye, où Henri Hubert assurait la promotion de la sociologie durkheimienne. Malheureusement, l'école de Saint-Germain fit long feu: la Grande Guerre, la mort de Déchelette, puis celle de Durkheim en 1917 et enfin la disparition de Hubert en 1927 éteignirent les velléités de rénovation des antiquités nationales, tandis que les disciplines historiques se ralliaient à l'École des Annales. Il n'empêche que la forte impression produite par l'école historique sur Déchelette se ressent dès le troisième tome du Manuel, qui est consacré au premier âge du Fer. Se distinguant des volumes précédents, celui-ci s'ouvre sur une étude érudite de la tradition historiographique, à laquelle le protohistorien accorde manifestement le plus haut intérêt et même la plus grande confiance ${ }^{14}$. Ce parti pris contribue sans doute à mettre le Manuel en porte-à-faux et à le desservir aux yeux des archéologues comme des historiens. De plus, si les références et les séries typologiques méritent toujours l'intérêt et l'indulgence des spécialistes, les développements historiques et théoriques apparaissent bien vieillis. Ce discrédit est d'autant plus regrettable qu'avant Déchelette, la protohistoire française n'était ni structurée, ni même reconnue en tant que telle; si elle finit par l'être au bout

12 S. Lewuillon, “Destins d'estampes. L'image archéologique dans la seconde moitié du XIX ${ }^{e}$ siècle en France", in Actes du Colloque Napoléon III et l'archéologie, Compiègne, 14-15 octobre 2000, Bulletin de la Société historique de Compiègne 37 (2001), p. 49-70 ; "Archaeological Design : a new Development in $19^{\text {th }}$ Century Science", Antiquity 76, 291 (2002), p. 223-234.

13 Les recherches préalables et le premier jet de l'ouvrage ont été réalisés partiellement dans le cadre d'une mission de l'INHA (avec le soutien d'AREA II). Les recherches complémentaires ont été poursuivies au cours de deux sessions des Techniques de l'Archéologie en Europe (anciennement à l'université d'Artois, aujourd'hui à l'université de Picardie Jules Verne). Des compléments ont été apportés avec le concours de BIBRACTE lors de la constitution d'un dossier de numérisation des archives de l'archéologie (projet NUMICON - MRT, ministère de la Culture, 2003-2004: S. Lewuillon, "Projet pour un ouvrage illustré: Le Musée de papier (titre de travail)”, Bibracte, 2004). 
d'une longue gestation, c'est à lui que nous en sommes redevables, ainsi qu'aux travaux de tous horizons qu'il cita dans ses notes infrapaginales.

Le mérite principal de Déchelette est d'avoir conçu l'archéologie unifiée méthodologiquement comme une science qui ne fût plus une annexe de l'histoire, mais une autre façon d'écrire celle-ci. En revanche, du point de vue de l'archéologie moderne, le bilan du "terrain » est plus mitigé. Pour le conservateur de Roanne, ces données matérielles devaient servir à conforter les chronologies et les typologies de l'aire celtique. Il joignait à cela une conception de l'archéologie comparée issue de ses premiers travaux d'histoire de l'art, où l'interprétation évolutionniste des objets avait été définie comme l'outil principal de l'analyse stylistique. Cette doctrine, mise à l'épreuve dans le volume de Hallstatt, s'épanouit dans celui de La Tène, formé d'un catalogue d'objets encore plus considérable que le précédent, qui sert à mettre en perspective les milieux et les modes de vie des populations celtiques: c'est le lieu d'une ethnographie comparée qui ne dit pas son nom, mais où perce l'influence de Hubert et des ambitions comparatistes qu'on a évoquées plus haut. Tous les systèmes chronologiques qui sont en usage aujourd'hui dans les études protohistoriques sont fondés, à quelques nuances près, sur ce principe qui, bien que perfectionné par l'informatique, n'a jamais été fondamentalement renouvelé. L'intuition de Déchelette le lui fit pressentir à une époque où prévalait encore l'empirisme, mais l'essentiel pour lui était d'abord de l'élever de l'échelle locale à celle d'un continent. Après la guerre, et donc après Déchelette, un nouvel effort d'unification eût été nécessaire, mais la chronotypologie ne rencontrant que peu de succès en France, la méthode de Déchelette finit par se perdre dans l'étroitesse d'esprit qui marqua l'entre-deux-guerres.

C'est à partir de ces considérations qu'il convient d'entreprendre le bilan, car le volet protohistorique du Manuel est bien le dernier de la main de Déchelette. Certes, les grandes lignes du tome gallo-romain sont connues: elles n'annonçaient pas de grands bouleversements. Plus scrupuleux que jamais, Déchelette avait recueilli une masse impressionnante d'informations de première main et mis toute la France archéologique en fiches, en photographies et même en cartes postales. On dispose avec ces deux derniers types de documents d'un ensemble considérable, qui demanderait d'être classé selon les principes de la conservation des matériels photographiques et de l'inventaire patrimonial, véritable jardin secret de Déchelette ${ }^{15}$. Pas de bouleversement, mais peut-être une nouvelle ambition. On croit percevoir dans ce nouveau programme le désir de mieux s'en référer à la notion de civilisation en en reconnaissant les contours, c'est-à-dire les marqueurs archéologiques de l'identité culturelle. Cette nouvelle orientation conduisait l'archéologue à dépasser l'étude analytique des mobiliers pour se

15 S. Lewulllon, "Garder, regarder, voir et savoir. Le statut de l'image archéologique au XIX ${ }^{\mathrm{e}}$ siècle en France". Images et imagerie, 132 e congrès du CTHS, Arles, 16-20 avril 2007 ; "Positif/Négatif. Les antiquités nationales, l'estampe et la photographie", Les Nouvelles de l'Archéologie 113 (septembre 2008), p. 37-45. 
tourner vers les archives du sol. C'est de la fouille que Déchelette espérait la vérité sur les Gaulois. Il avait choisi pour cela le lieu emblématique qui lui avait suggéré naguère ses plus fécondes hypothèses, dont celle de Stradonice: Bibracte. Délaissant Roanne pour la première fois de sa vie, le neveu de Bulliot entreprit durant l'été de 1914 de s'installer en tête à tête avec sa documentation dans une demeure isolée à proximité du Mont-Beuvray. Un programme enfin résolument archéologique, mais qui ne verrait jamais le jour: Déchelette n'avait plus que trois mois à vivre.

À n'en pas douter, sa fin prématurée a privé l'archéologie nationale d'une refondation comparable à celle que Marc Bloch et Lucien Fèbvre devaient offrir quelques années plus tard aux études historiques. Pourtant, malgré l'ouverture immense qu'annonçait son œuvre, le bilan de Déchelette ne fait toujours pas l'unanimité. Peut-être faut-il en chercher la cause dans son ultime évolution intellectuelle. Se fâchant avec les préhistoriens, le maittre de la protohistoire s'était rapproché de celui de l'historiographie gauloise, Camille Jullian. Or, ce dernier était aussi décrié par les archéologues français que par les savants étrangers, en raison de son obstination à méconnaître l'archéologie (disqualifiée au profit des textes), de ses futiles obsessions chronologiques (susceptibles de remettre en question l'existence de l'âge du Bronze) et finalement de son incrédulité envers le concept même d'une "Europe de l'âge du Fer ». Avec un tel patronage, Déchelette s'avouait-il dépassé par une archéologie qui se faisait ou se défaisait désormais sans lui ou bien était-il, dans ces circonstances terribles de 1914, un déçu de l'histoire? En tout cas, il avait pressenti sa fin, s'avouant rongé par la perspective de trouver dans les tranchées adverses ceux avec qui il avait partagé la même ferveur intellectuelle pour le passé de l'Europe. Quoi qu'il en soit, le doute ultime qu'il a éprouvé constitue un avertissement pour les lecteurs de ses archives: dans la biographie du maître de Roanne, ce n'est pas aux péripéties qu'il convient de s'attarder, mais aux leçons d'histoire.

Serge LewUiLlon

Techniques de l'Archéologie en Europe

\& Valorisation des Patrimoines en Europe

Université de Picardie - Jules Verne

serge.lewuillon@u-picardie.fr 